\title{
Les pseudonymes des internautes camerounais : quels procédés et quelles visées communicationnelles?
}

\author{
Venant Eloundou \\ Université de Yaoundé, Cameroun \\ evenant2002@yahoo.fr
}

\begin{abstract}
Résumé. Mettant à contribution les principes de l'analyse du discours et de la nomination, cette étude examine les pseudonymes adoptés par les internautes camerounais qui animent les débats sur la toile. Le postulat de base est que même si le fonctionnement de la pseudonymie sur Internet est régi par des caractéristiques générales, il est aussi nécessaire de l'étudier en tenant compte de l'univers socio-discursif où elle est construite, afin de voir comment ces caractéristiques s'adaptent aux différents contextes. Dans cette optique, sont scrutés, les procédés de construction des pseudonymes par les Camerounais de la diaspora et ceux qui résident au pays. Les mécanismes les plus saillants sont: les patronymes pseudonymiques, les indicateurs culturels, l'art, le sport, la toponymie, les facteurs historiques, politiques, idéologiques, philosophiques et l'ethos des internautes. L'étude démontre que la significativité de ces pseudonymes est l'expression des identités, de la culture et des visions sociopolitiques. Internet offre ainsi un espace de dialogue ou l'identité réelle des acteurs est cryptée. Mais ces masques n'excluent pas la saisie des différentes intentions de communication des internautes.
\end{abstract}

\begin{abstract}
Pseudonyms of Cameroonian Internet Users: Which process and for which communication aims?

Drawing on the principles of speech analysis and naming, this study examines the pseudonyms of Cameroonian Internet users who animate debates on the web. The basic assumption is that even though the functioning of screen names on the Internet is governed by general characteristics, it is also necessary to study it taking into account their socio-discursive universe, in order to see how these characteristics adapt to different contexts. As a matter of fact, the methods of construction of pseudonyms by the Cameroonians of the diaspora and those who reside in the country are examined. The most prominent mechanisms are: screen surnames, cultural indicators, art, sport, toponymy, historical, political, ideological, philosophical factors and the ethos of Internet users. The study demonstrates that the significance of these screen names is the expression of identities, culture, and socio-political visions. Thus, the Internet offers a space for dialogue where the real identity of the actors is encrypted. But
\end{abstract}


these masks do not prevent the reader from discovering the various intentions of communication of Internet users.

\section{Introduction}

Les technologies de l'information et de la communication favorisent des pratiques susceptibles d'intéresser les chercheurs en sciences du langage. Dans cette optique, les pseudonymes des bloggeurs et des internautes ont été largement étudiés. Nous pouvons noter l'étude d'Anis (2001) basée sur la sémiotique des représentations de l'ego dans la communication par ordinateur. L'auteur met en exergue les différents procédés mobilisés par les internautes pour s'auto-nommer. Parmi ces procédés, il y a des pseudonymes, des avatars, des formes graphiques personnalisées. L'auteur conclut que ces paramètres participent à la mise en place du dialogisme interactionnel et la manifestation d'un ethos discursif. Georgeta Cislaru (2009) aborde les pseudonymes des internautes comme un fait discursif dont le fonctionnement est régi par le contexte social, le cadre thématique et l'identité personnelle (2009: 2). Les valeurs magiques des pseudonymes sont examinées par Maribel Fehlmann (2010). Elle propose une typologie fondée sur le contenu des pseudonymes. Ce qui l'amène à conclure que le choix des autonymes tient compte «d'une possible croyance magicoverbale chez certains internautes » $(2010: 274)$. C'est le cas des pseudonymes encomiastiques, conjuratoires, comminatoires, bénédictifs, etc. Anne-Sophie Béliard (2009) analyse les fonctions primordiales des pseudonymes à partir des discours émis par certains participants au forum de fans de la série télévisée Prison Break. Elle identifie deux principales fonctions : la fonction de présentation et de positionnement et la mise en exergue de l'image de soi. L'étude de Marcienne Martin (2013) va s'appesantir sur l'opposition entre la nomination dans la société virtuelle et la société réelle. A partir des résultats d'une enquête menée, l'auteur pose les différents statuts des pseudonymes sur Internet, selon le degré de rapprochement avec la nomination dans la société réelle. Par ailleurs, il propose une distribution des pseudonymes par champ sémantique. Khaled Slama (2011) s'intéresse à la distinction du pseudonyme au patronyme, au prénom, au surnom, au nom commercial. Selon cet auteur, le pseudonyme est « un masque de l'identité réelle, il sert à camoufler, à cacher, en somme à la garder secrète ».

Dans ces conditions, la pseudonymie sur Internet est différente de la cryptonymie, puisque l'internaute ne crypte pas son véritable nom, de telle manière qu'il soit reconnaissable par des proches ou des connaisseurs. Par ailleurs, Khaled Slama met l'accent sur les fonctions des pseudonymes et les aspects juridiques. Ces différentes études, parmi tant d'autres, analysent globalement les pseudonymes, en insistant sur leurs mécanismes langagiers, leur typologie et leur sémiotisation discursive. A la suite de ces différentes orientations, nous nous proposons de mener une étude contextualisée axée sur une catégorie sociale des internautes ayant des déterminants contextuels spécifiques. Il s'agit d'interroger les jeux et les enjeux de l'auto-nomination des internautes camerounais qui animent des forums de discussion sur certains sites Internet.

La réflexion repose sur deux questions fondamentales: Quels sont les procédés pseudonymiques qui président à la nomination des membres des sociétés virtuelles camerounaises ? Quels sont les visées qui en découlent? Après avoir esquissé le cadre méthodologique et théorique de l'étude, nous nous attellerons à analyser les mécanismes pseudonymiques mobilisés avant de déboucher sur leurs rendements communicationnels. 


\section{La posture méthodologique et théorique de l'étude}

En sociolinguistique, la délimitation du terrain d'étude est fondamentale, dans la mesure où c'est ce dernier qui donne toute la pertinence à une analyse. Le terrain est donc le ferment de la sociolinguistique. Ainsi, pour Boutet, cité par Pierozak (2007 : 8-9), « la sociolinguistique peut être considérée comme une forme de linguistique de terrain. Elle ne peut s'exercer sans avoir recours à des observations de situations sociales effectives, quelle qu'en soit la nature : espaces publics, familles, réunions associatives, situation de travail, écoles, etc. Les données sont recueillies dans des situations sociales réelles ». Toutefois, il convient d'opérer un dépassement pour cerner le terrain comme un cadre d'intervention du chercheur pouvant être virtuel (comme Internet) et duquel il tire les observables de son analyse. L'espace virtuel de l'Internet devient une forme de situation sociale réelle, à la seule différence qu'il exclut le contact direct. Mais certains paramètres observés dans des situations effectives peuvent être symétriques aux situations virtuelles. La dichotomie qui caractérise la notion de terrain entraîne ainsi sa complexité. Il peut arriver que les terrains ne soient pas «des lieux objectifs et extérieurs au chercheur». Le chercheur construit son terrain en fonction des objectifs assignés. Dans ces conditions, Pierozak (2007: 9) fait valoir que « le terrain désigne l'ensemble des contraintes qui pèsent sur la construction des phénomènes pertinents pour le chercheur ». Pour Pierozak (2007: 8), Internet est devenu un terrain complexe pour l'analyse des pratiques langagières, des représentations linguistiques et de l'analyse de discours.

Le terrain de l'étude est Internet. Nous y avons exploré trois forums de discussions hébergés dans les sites suivants: www.forumbonaberi.com, www.cameroon.info.net et www.camer.be. Le premier site a la particularité d'être un espace de débats engagés par des bloggeurs. La stratégie consiste à initier une discussion sur un aspect en lien avec le Cameroun. A cet égard, les thèmes suivants ont suscité des débats généralement polémiques : Les motes ki ne kno pas spik le camfran (les gens qui ne savent pas s'exprimer en camfranglais), Pourquoi les gens de doul ne kno pas spik le camfran? (Pourquoi les gens de Douala ne savent pas parler camfranglais?), Whitiser le camfranglais ou le patois (Parler le camfranglais le patois (langue nationale ou maternelle) avec un accent du français hexagonal), Dico camfranglais (Dictionnaire camfranglais), Et la langue est. Pour ce qui est des deux derniers sites, les débats sont consécutifs aux articles publiés et postés sur le même site. Les internautes réagissent à la suite de la publication d'un article soit dans un journal camerounais, soit sur ce site. Plusieurs articles publiés dans certains journaux écrits et relayés sur www.cameroon.info.net ont stimulé les discussions : Fran Anglais : New language for divided Cameroon, Rigobert Song: la prochaine coupe du monde est déterminante pour sa carrière, Diaspora et développement: le point de vue d'Alain Anyouzoa, Dialectes : langues maternelles pour le développement, Le pays est sucré (par Alex) : "Mes frère, arrêtons de whitiser, moi je dis qu'il faut camerouniser " et CamerounFête nationale du 20 mai 2014: Barack Obama écrit à Paul Biya. Nous avons retenu plusieurs thèmes dans www.camer.be, qui ont donné lieu à des débats auxquels les internautes ont pris part. On a entre autres : Journée africaine de François Hollande : un retour sibyllin aux bases militaires, Pétition pour la fin de toute coopération entre la France et l'Afrique francophone, Aide au développement : la France écarte le Cameroun, etc. L'enjeu n'est pas d'analyser, de manière quantitative, les nominations des internautes. Il s'agit de nous intéresser particulièrement à certains cas significatifs. Le choix des sites se justifie par le fait que notre objectif est d'analyser la pseudonymie dans un cadre contextuel, notamment une forme de praxis propre à un groupe social (les Camerounais de la diaspora qui interagissent sur la toile avec ceux qui sont au pays). Nous avons opéré les 
choix des autonymes en fonctions de leur pertinence. Ce qui revient à dire que nous priorisons la compréhension et la significativité.

Quant à l'approche théorique, la réflexion repose sur les principes de l'analyse du discours, sous-tendus par l'approche conceptuelle de nomination élaborée par Siblot (2001 et 2006) qui s'inspire des réflexions de Kleiber (1984). Pour Siblot les termes dénomination et nomination sont symétriques dans des usages ordinaires et même scientifiques ; pourtant à les examiner, ils ne signifient pas la même chose. A cet égard, il sonde leur différente étymologie. L'examen subtil de ces deux concepts le conduit à démontrer que la dénomination se distingue de la nomination dans la mesure où la première relève de la langue portant sur un état stabilisé du processus de nomination, tandis que la seconde est du domaine du discours. Elle est un processus et non un état stabilisé. L'acte de nommer convient à la nomination et l'acte de dénommer est lié à l'aboutissement du premier. Dès lors, « nommer, c'est nommer quelque chose, d'une façon intrinsèquement référentielle qui comporte un choix réaliste » $(2006: 38)$. Nous souscrivons à la distinction faite par Siblot et considérons les pseudonymes analysés non pas comme des dénominations, mais comme des nominations discursives. Ces nominations sont scrutées dans une perspective de l'analyse du discours.

L'analyse du discours, dans une perspective de l'école française et suisse, a été largement développée ou conceptualisée. Maingueneau (2016) et Barry (2002) en font une synthèse exhaustive. Ils montrent que l'analyse de discours est diversement appréhendée et appliquée aux différents domaines. Dans le cadre de cette étude, nous la considérons comme une approche d'exploration de discours dont l'«objet n'est ni l'organisation textuelle ni la situation de communication, mais ce qui les noue à travers un certain dispositif d'énonciation (Maingueneau, 2012: 4-5). L'étude ne prend pas appui sur des unités topiques prédécoupées par des pratiques verbales (même si par ailleurs on peut postuler que les sociétés virtuelles au sens de Martin (2013) donnent lieu à un genre de discours et de positionnement), mais sur des unités construites par nous-même à partir d'une catégorie topique considérée: les discours des internautes de trois forums de discussions. Cette catégorie d'unités a la particularité «d'impliquer beaucoup plus fortement le chercheur qui [...] construit ses corpus à partir des catégories topiques, autour de points de fixations qui ne sont pas des domaines découpés par les pratiques sociales » (Maingueneau, 2012: 8). Nous avons construit le corpus d'étude en sélectionnant les formes d'auto-nomination des internautes pertinentes pour le problème d'étude, puisque " l'analyse du discours, sans se prétendre critique au sens habituel du terme, repose sur des évaluations, par la sélection même des énoncés qu'elle étudie » (Maingueneau, 2012:13). La dimension discursive adoptée nous conduit à privilégier une démarche herméneutique visant à « dévoiler, à dé-couvrir ce que les textes sont sensés cacher». Toutefois, il s'agit là d'une herméneutique «sombre » qui donne la possibilité d'analyser «toutes les sortes de textes, y compris les échanges les plus familiers. L'interprète, par son analyse, montre qu'il est supérieur aux textes qu'il étudie. L'analyse, au lieu de rendre le texte plus énigmatique, réduit la profusion du sens » (Maingueneau, $2012: 12$ ).

\section{Les constructions de la pseudonymie}

Pour des besoins de la sécurité et d'accès à certains forums de discussion sur Internet, l'adoption d'un pseudonyme est une exigence. L'internaute construit ainsi une autre identité virtuelle. Dans ce cadre, il est incontestable que les autonymes ne relèvent pas de ce que Siblot appelle un acte de baptême, par conséquent ils demeurent un fait de discours et ne peuvent pas aboutir à la dénomination qui est le résultat du processus de la nomination. 
Dans cette section, il convient de cerner les constructions des pseudonymes dans le corpus d'étude, selon une approche taxinomique.

\subsection{Les pseudonymes patronymiques}

L'un des mécanismes nominatifs est l'usage des patronymes inhérents aux aires culturelles camerounaises. Les participants aux forums de discussions utilisent des patronymes. C'est le cas de EKOBENA (Les motes ki ne kno pas spik le camfran, www.forumbonaberi.com), EDGAR FOTSO BILOA (Douala, 20 février 2007, Francis Ngwa Niba, Correspondance, "Fran Anglais: New language for divided Cameroon", www.cameroon.info.net) et ESSENGUE (France Afrique, pétition pour la fin de toute coopération entre la France et l'Afrique francophone, Correspondance de P. Ludovic Lado, 23/06/2014) :

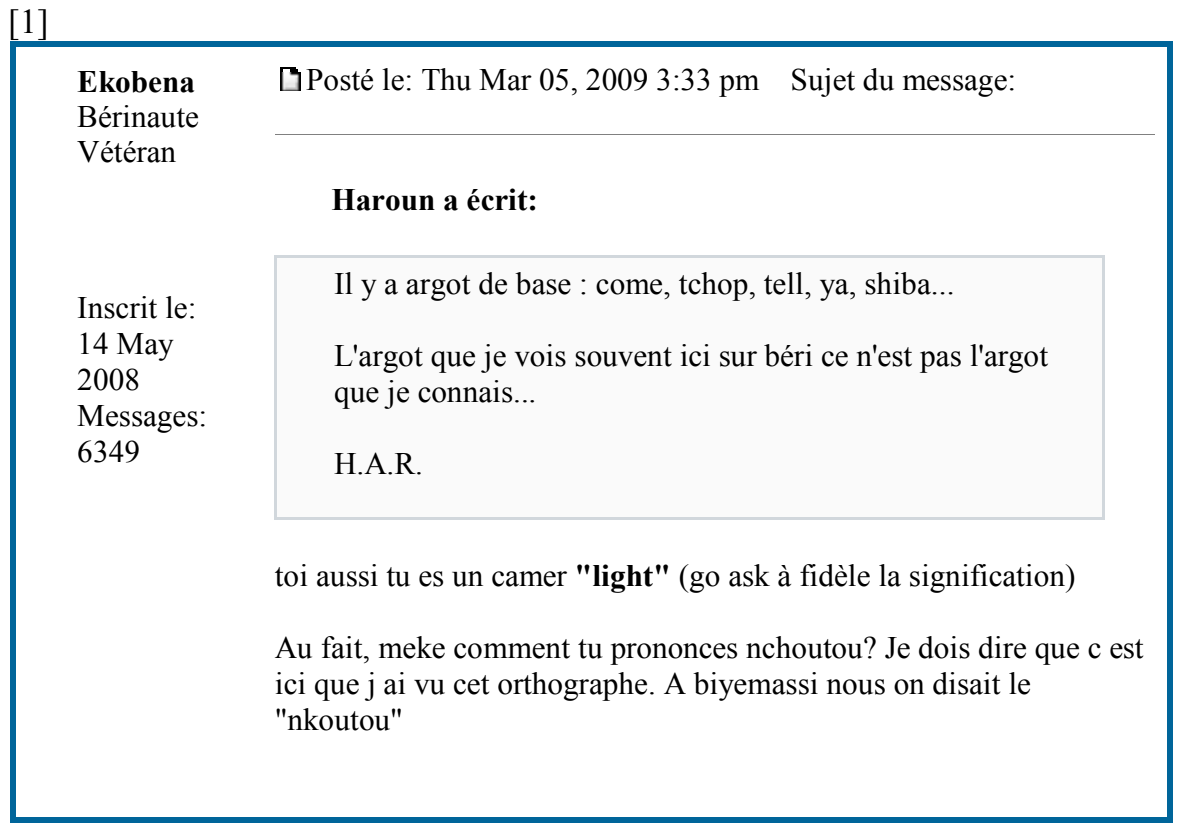

[2]

\section{Essengué}

(a) ludoviclado ceux à qui tu demandes de signer la pétition ne le feront pas, tout simplement parce qu'il y a un problème de foi en dieu et de prise de conscience nous qui n'avons pas de chance d'être comme des dirigeants des pays d'afrique, alors que nous avons la foi et l'amour pour nos frères d'afrique, nous devons seulement leur écrire, soit leur parler par des médiats, afin de les sensibiliser sur ce qu'ils devraient faire pour atteindre la liberté et la prospérité africaine..

[3]

Edgar FotsoBiloa

23-Feb-2007 21:30

Sous Le Baobab

\section{EST}

Laura

Carla

(Canada)

STP, encore un diva. L'histoire de Tatoo le gardien de but a failli me kill. Encore un diva ressé. Moi je te promets une jong. Kesque tu manges ? Ton goût ne peut pasme dépasser. Raconte alors ressé. I di yafromyou. 
Ces trois pseudonymes reflètent l'univers camerounais. Il s'agit là des patronymes que l'on rencontre au Cameroun et qui sont portés par des Camerounais. Le premier (EKOBENA) et le deuxième (ESSENGUE) sont fortement marqués dans les régions du Centre, du Sud et de l'Est. Le troisième (FOTSO BILOA) constitue une nomination lexicalement composée de deux origines ethniques : bamiléké et beti.

\subsection{Les pseudonymes culturels et claniques}

Généralement, dans la plupart des sociétés de l'Afrique noire, certaines désignations dénotent le statut social des personnes. Il peut s'agir de la hiérarchisation sociale, du statut matrimonial, du statut de la procréation. Au Cameroun, certaines ethnies utilisent ces dénominations qui permettent de distinguer les personnes vivant dans une communauté. Les internautes utilisent ces formes pour s'auto-nommer. A ce titre, nous avons : MAGNE (Dico Camfranglaiswww.forumbonaberi.com), PA'A TAGNE (Guinée EquatorialeFrance: M. Obiang Jr privé de ses belles voitures par la justice, Béatrice Gurrey, Lemonde.fr, www.camer.be, 23/11/2012), NKUNKUMA 2 (L'affaire franco-africaine du Mali : la triple humiliation, correspondance de Ludovic Lado, www.camer.be, 10/02/2013) et BETIBENANGA (Succession "tribale" de Paul Biya: Le Professeur Hubert Mono Ndjana accusé, se défend avec vigueur, Yaoundé, 25 Mars 2014, Ouest Littoral, www.cameroon.info.net) :

\begin{tabular}{|c|c|}
\hline \multirow{2}{*}{$\begin{array}{l}\text { MAGNE } \\
\text { Ancien }\end{array}$} & D Posté le:SatFeb 27, 2010 4:39 am Sujet du message: \\
\hline & $\begin{array}{l}\text { heureusement ke Elan est kem t'expliker les ways de son langage ci. } \\
\text { Petitbandit peutr elle est brune fallala un peu ca pe gui }\end{array}$ \\
\hline $\begin{array}{l}\text { Inscrit le: } \\
12 \text { May } \\
2008 \\
\text { Messages: } \\
16781\end{array}$ & \\
\hline
\end{tabular}

[5]

\section{PA"A TAGNE (Avenches) SWITZERLAND}

les zenfantsoohh!!

laneijeudeu la télé vu o cameroonnaipa froid, et sai bon.

doncqueuseuluikicroa queue le yeurope ai leu paradineuconait rien.

sa va aitre vendu o zenfers,le plu zofrant et maime le kopé ou leu rang fabius ou filou va racheter sà là pour un francksymbolik.peut aitre maimesarkozyzy a déjacomenssé le négosiassion pour acheter leu richesse là,comeu pour leu libie.

[6]

\section{NKUNKUMA2 (Los Angeles) UNITED STATES}

humiliation?fallait 'il laisser les fous de dieu continuer a couper les mains et les pieds au gens ou les

lapider?

pardon. qu'on laisse les gens tranquille avec les fausses theories d’un panafricanisme qui n`a ni tete ni queue... 


\section{BETIBENANGA}

03-Apr-2014 11:09

EDT

Ngulmekong

Cessons de regarder le Cameroun comme une réalité statique. Concevons notre pays comme une réalité évolutive.

Ce préalable posé nous oblige raisonnablement à considérer la tribu comme élément historique qui, actuellement, ne peut être un vecteur de la construction nationale que lorsque les valeurs qu'elle véhicule, rencontrent un écho dans la diversité culturelle qui est la notre. Il s'agit donc ici, de trouver à chaque fois, le plus grand dénominateur commun à nos différentes tribus, pour l'érigeren réalité culturelle établie et même codifiée.

Les quatre nominations proviennent de l'univers social du Cameroun. Les deux premières relèvent des titres distinctifs inhérents à la région de l'Ouest Cameroun. Elles réfèrent respectivement à une femme qui a mis au monde des jumeaux et à un homme, père de ces jumeaux. Il s'agit donc d'un titre pouvant être utilisé, dans certaines circonstances, en lieu et place du patronyme ou du prénom. Pour ce qui est du troisième cas, (NKUNKUMA 2), il est lié au système de l'organisation coloniale de la société beti. C'est une appellation des chefs traditionnels institués par le colonisateur et servant d'intermédiaires entre les populations et l'administration coloniale. En (7), nous avons un hypéronyme: BETIBENANGA désignant un macro-groupe composé de plusieurs tribus (ewondo, bulu, eton, menguisa, mvélé, yebekolo, etc.). Selon certaines sources orales, ce groupe aurait pour ancêtre Nanga (thèse discutable) et aurait pour descendants : kolobeti, eton, mvele, bulu, ntumu, bene, fong, Menguisa, etc. Par ailleurs, certains indicateurs culturels à caractère discursif et viticole participent à la pseudonymie :

[8]

$\begin{array}{ll} & 25- \\ \text { MAN TARA } & \text { May- } \\ \text { Chicago } & 2014 \\ & 12: 52 \\ \text { ED }\end{array}$

En dehors $\mathrm{du}$ fait que ce mec porte un maillot des lions... $\mathrm{Au}$ fait je n'ai pas compris un seul mot. Il aurait aussi bien pu chanter en "kasakstanis". Enfin, je suis pour la diversification du paysage camerounais. Je crois qu'on va devoir commencer aimporteter des tas de peuples, chinois, indiens, europeens, amerindiens, etc. Nous sommes trop camerounais. Va falloir dilluer un peu la purete. Justement le Cameroun risque deja de mourir de purete (Yaoundé, 21 mai 2014, Alex, Correspndance, «Cameroun-Musique Vidéo: Le pays est sucré (par Alex) : «Mes frères, arrêtons de whitiser, moi je dis qu'il faut camerouniser », 25/05/2014, www.cameroon.info.net

[9]

MATANGO (Noranda) CANADA

bientôt, on va perquisitionner chez crabe d'okola, notre receleur de biens mal acquis (France: François Hollande, «je veux définitivement rompre avec les dérives de la Françafrique », Christophe Courtin, Le Messager, 15/02/2012, www.camer.be).

MAN TARA est une expression qui relève de la langue ewondo (une langue camerounaise, classé à A72a par Guthrie, 1958/1967). Cette formule est une appellation ou un vocatif permettant d'interpellé un frère de même père au sens restreint ou alors un cousin paternel. MATANGO désigne le vin extrait du palmier à huile prisé non seulement 
par les habitants des zones rurales, mais aussi ceux des zones urbaines qui se retrouvent dans des espaces marchands populaires.

\subsection{Les noms des vedettes}

L'un des topiques pourvoyeurs des pseudonymes est le domaine des stars. En effet, certains bloggeurs portent les noms des footballeurs. C'est le cas de : ARANTES et BEBETO :

[10]

\section{ARANTES}

Yde

04-Jul-2008 09:21 EDT

brillant intellectuel sauf que je connais un camerounais qui a coha $\% \% \%$ avec lui et a qui il a fait paye tout le loyer en arguant qu il 1 aidait alors qu il profitait de son innocence changer l'afrique c'est commencer par etrehonnete envers son compatriote aussi faible soit il (Jean Bruno Tagne, LeJour ; "Rigobert Song : La prochaine coupe du monde est déterminante pour sa carrière », www.cameroon.info.net)

\section{[11]}

\section{BEBETO FRANCE}

voyez-vous!

quelle image le cameroun donne lorsque ses athlètes se fondent dans la nature à londres? quelle signification d'après vous cela donne,

eh bien c'est simple à comprendre par le commun des mortels:

- la vie n'est pas aisée chez-nous au cameroun

- on préfère errer n'importe où en europe que de retourner au cameroun (Cameroun : Que se passe-t-il entre Paul Biya et les Présidents français? Michel MichautMoussala, Aurore Plus, www.camer.be).

Ces pseudonymes peuvent être endogènes ou exogènes. ARANTES est le surnom d'un footballeur camerounais très talentueux: Grégoire Mbida, premier buteur de l'Afrique noire subsaharienne à une coupe du monde (Italie 1982) lors d'un match qui opposait le Cameroun à l'Italie. Toutefois, ARANTES porté par Grégoire Mbida peut être considéré comme un cryptonyme, étant donné que des proches ou des connaisseurs savent qu'il s'agit bel et bien de Grégoire Mbida et non du footballeur brésilien Edson Arantes do Nascimento, connu sous le nom de "Roi Pelé ». BEBETO constitue une occurrence exogène servant à nommer un internaute camerounais. Ce nom adopté par cet internaute de camer.be est inhérent au footballeur brésilien, vainqueur de la coupe du monde de 1994, organisée aux Etats-Unis. L'on retrouve aussi les cas de nominations tirés de l'art musical et de la littérature :

[12]

$\begin{array}{ll} & 23- \\ \text { WANGRIN122 } & \text { May- } \\ \text { Bonn } & 2014 \\ & 10: 01 \\ & \text { EDT }\end{array}$

Un jour avec des potes on lappait un pater en frankanglais dans le metro. $\mathrm{Au}$ moment de descendre un pote se tourne vers et le pater et dis est ce que tu amemeya. Et 
le pere de repondrebindjiment!!! (Yaoundé, 21 mai 2014, Alex, Correspndance, «Cameroun-Musique Vidéo: Le pays est sucré (par Alex) : «Mes frères, arrêtons de whitiser, moi je dis qu'il faut camerouniser », www.cameroon.info.net, 23/05/2014)

\title{
[13]
}

\begin{abstract}
ZANGALEWA(Halle) BELGIUM
ludoviclado,

même si tu n'es pas assez tranchant, je te souhaite néanmoins la bienvenue dans le club de ceux qui pensent que nous africains sommes responsables de nos turpitudes et ne devons nous en prendre qu'à nous-mêmes, à cause de l'ignorance et de l'obscurantisme dans lesquels nous nous complaisons (L'affaire franco-africaine du Mali : la triple humiliation, correspondance de Ludovic Lado, www.camer.be, 10/02/2013).
\end{abstract}

WANGRIN est le nom d'un personnage du romancier malien Hampâté Bâ, dans son roman intitulé L'étrange destin de Wangrin, personnage qui se distingue, tout au long du récit, par la gloire et l'humanisme à l'endroit des pauvres à l'époque coloniale. ZANGALEWA désigne une chanson camerounaise qui est du répertoire militaire. Cette chanson avait été composée et chantée par un groupe de militaires camerounais musiciens en 1986, groupe appelé Golden Sounds. Il faut préciser que cette expression est issue d'une modalité phrastique de la langue ewondo. C'est la transformation de zaangaloéwà (qui t'avait appelé dans le corps militaire ?).

\subsection{Les désignations toponymiques}

Les indices toponymiques participent aussi à la construction des pseudonymes des internautes. Il s'agit prioritairement des toponymes camerounais :

[14]

\section{NDOKOTI-MVOGMBI \\ Etoudi Presidence}

$$
\begin{aligned}
& \text { 30-May- } \\
& 2008 \\
& 11: 14 \\
& \text { EDT }
\end{aligned}
$$

Conceptman

Rire.

Je t'ai YA MO sur ce TOLI (Jean Bruno Tagne, LeJour ; « Rigobert Song : La prochaine coupe du monde est déterminante pour sa carrière », www.cameroon.info.net) [15]

\section{DEÏDO1}

Principauté De Deïdo

10-Jul-2008 00:56

EDT

Tna,

je ne cherche pas d'histoire, est-ce qu'on peut parler de tout? pourquoi ne peut-on pas parler des Bamilélés? «je ne vois pas quelle tribu peut jeter la pierre sur une autre $\mathrm{Ce}$ n'est pas le but...Et personne même pas moi n'a dit qu'il était meilleur que d'autres. Ce que tu parles de la Guiness Cameroun est un caractère propre aux Bamiléké et c'est ce que je dénonce (Ferdinand Mayega, Le Combattant, Correspondance, «Diaspora et développement : le point de vue d'Alain Anyouzoa », www.cameroon.info.net)

\section{[16]}

\section{MVOG-ADA}

je note,et c'est pour m'en réjouir queles 3500 soldats français affectés dans dans la zones sahélo-saharienne pour la lutte antiterroriste ne seront pas du tout déployés au cameroun mais d'après yves le drian en centrafrique,auniger au tchad,aumali,et au burkina;car ces gens-là,quand, ils mettent les pieds quelque part,c'est pour y semer plus de 
désordres qu'ils n'en ont trouvés;restons prudents parce qu'il (Journée africaine de François Hollande : un retour sibyllin aux bases militaires, MunaDimbambe, Aurore Plus, www.camer.be)

\section{@ KOLOFATA}

hollande n'a jamais été ministre, informez-vous avant de débiter vos inepties.il a été député-maire, conseiller général à tulle en corrèze

mercibien.

KOLAFATA(Yaoundé) CAMEROON $₫$ (France-Cameroun: François Hollande est-il au courant de ce qui se passe au Cameroun ? Issa Behala, wwwcamer.be)

En lieu et place des patronymes pseudonymiques, certains internautes préfèrent les noms des localités du Cameroun. NDOKOTI-MVOGMBI est un couple toponymique composé de deux noms des quartiers populeux des villes de Douala et Yaoundé. DEIDO1 renvoie à un quartier de Douala. Pour ce qui est de KOLOFATA, il réfère à un chef-lieu d'arrondissement. Il s'agit d'une ville située à l'Extrême-Nord du Département de MayoSava, et à la frontière du Cameroun avec le Nigéria. Cette ville est le théâtre depuis quelques années, des exactions terroristes de la secte islamiste Boko Haram.

\subsection{Les références historiques, philosophiques et mythologiques}

Il arrive que certains internautes utilisent des références de l'histoire africaine, la mythologique et la philosophie pour s'auto-nommer. Ce procédé s'illustre par des indices tels que CLEOPATRE (Whitiser le camfranglais ou le patois, www.forumbonaberi.com), EURYALE8 (Dico Camfranglais, www.forumbonaberi.com) et PLATON (Et la langue est, www.forumbonaberi.com) :

[17]

CLEOPATRE \Posté le: Thu Nov 19, 2009 2:36 am Sujet du message:

Invité

Afreaka j'aime bien comment tu chantes, je voudrais te produire....

[18]

PLATON DPosté le: Tue May 01, 2012 1:24 am Sujet du message:

Petit

shabbaeur

Pour moi, tout celàreleve d'une utopie.

Au Cameroun en particulier, pour faire d'une langue locale une

Inscrit le: langue nationale, il y'aurait trop d'obstacles à surmonter (et si on

04 Nov estime avoir assez d'énergie pour les surmonter, c'est qu'il y'a un

2010 certain nombre d'autres problèmes à résoudre avant) :

Messages: - On a une multitude de langues nationales et même en procédant à

1148 de grands regroupements par langues visiblement "cousines" ex: douala-malimba, les langues peules, on devrait se retrouver au minimum avec 4 à 5 groupes linguistiques. Cette hétérogénéité 
complique sérieusement les choses (Et la langue est :

www.forumbonaberi.com)

\begin{tabular}{|c|c|}
\hline EURYALE8 & 口Posté le:SatFeb 27, 2010 12:51 am Sujet du message: \\
\hline $\begin{array}{l}\text { Inscrit le: } 27 \\
\text { Sep } 2008 \\
\text { Messages: } \\
4953 \\
\text { Localisation: } \\
\text { marcinelle }\end{array}$ & $\begin{array}{l}\text { crystal à force de lire ls gens écrire certains mots tu vas les } \\
\text { integrer; moi meme au debut je ne comprennais pas grd chose } \\
\text { mais today il yaqq mot que je comprend mm si dans la vie } \\
\text { courante je ne parle pas l'argot du pays. } \\
\text { On dit que les femmes sont les meilleures gardiennes de la } \\
\text { tradition, c'est dans le secret des cuisines que la culture passe } \\
\text { de mère en fille, on répète les gestes immuables et on } \\
\text { commente les dosages avec elle. (Dico Camfranglais } \\
\text { www.forumbonaberi.com) }\end{array}$ \\
\hline
\end{tabular}

CLEOPATRE est consécutif à l'art égyptien. Il s'agit du titre d'une comédie composée par Kamel Ouali et qui porte sur l'histoire de la dernière reine de l'Egypte antique. EURYALE renvoie à la mythologie grecque et romaine. Selon ces mythologies, il était un fils géant d'Ouranos (divinité personnifiant le ciel et l'esprit demiurge) et de Gaïa (déesse, mère de la race divine). Ce fils fut tué par la gigantomachie. Pour ce qui est de PLATON, il est un philosophe de la Grèce antique dont le fondement épistémologique est la Justice et le Bien commun de la cité.

\subsection{Les références socio-politiques}


Certaines internautes n'hésitent pas à utiliser les indicateurs socio-politiques pour s'autonommer. Ainsi en va d'EPERVIER, RDPC FOREVER, BIYABIVOLEUR et CEMAC6 :

[20]

\section{EPERVIER CANADA}

@mvog-ada on raconte que quelques soldats français sont stationnés à l'est au cameroun je n'ai aucune idée de leur nombre éventuel ni de leur puissance de feu (Journée africaine de François Hollande : un retour sibyllin aux bases militaires, MunaDimbambe, Aurore Plus, 23/06/2014, www.camer.be)

\section{[21]}

\section{RDPC FOREVER}

il faut sortir du franc cfa c'est la chaîne qui nous maintient en esclavage. coment expliquer que c'est un nigérian (dangote) qui vienne enfin faire concurrence au goroupe français lafarge (cimencam) ?

tchingangpouémi avait déjà essayé de le dire en son temps au camerounais il a été assassiné.

mpondo en 1976 a essayé de parlé du pétrole aux camerounais il a été assassiné avec toute sa famillle (Cameroun-France-aide au développement: la France écarte le Cameroun, Georges Alain Boyomo, Mutations, 01/08/2013, www.camer.be)

\section{BIYABIVOLEUR(Brussels) BELGIUM}

si la fanceafrique est révolu,il faudrait fermer toutes les bases militaires en afrique (France- Cameroun: l'encombrant monsieur Atangana, Jeuneafrique.com, www.camer.be, 13/09/2012).

\section{CEMAC6(Yaoundé) CAMEROON $\star$}

les riches là aussi dérangent. cessez d'aller construire chez les blancs !!!!!'afriqueà besoin de tout ces beaux batiments qui vont embellir nos villes !!!vous avez vu combien de leuco venir construire des batisses ici ? (France : François Hollande, « je veux définitivement rompre avec les dérives de la Françafrique», Christophe Courtin, Le Messager, www.camer.be 15/02/2012)

EPERVIER désigne une opération judicaire lancée en 2006, par le gouvernement du Cameroun, dont le but est de lutter contre la corruption qui sévit dans l'administration publique. Dans ce mouvement, plusieurs membres du gouvernement ont été interpellés puis incarcérés. Par analogie, l'épervier désigne cette machine qui fonctionne comme un rapace qui se jette sur les présumés détourneurs des fonds publics. RDPC FOREVER renvoie au parti politique au pouvoir, notamment le Rassemblement du Peuple Camerounais, qui a, à sa tête, le Président Paul Biya. L'internaute de l'énoncé [20] s'identifie par ce sigle.

Pour ce qui est de BIYABIVOLEUR, cette forme serait une déformation du nom du Président de la République du Cameroun dont la forme complète est Paul Barthélemy BIYA'A BI MVONDO. A la place de MVONDO, l'internaute a mis voleur. L'acronyme CEMAC (Communauté économique des Etats de l'Afrique Centrale), sert aussi de pseudonyme à l'internaute. 


\title{
2.7 Les désignations caractérisantes
}

L'un des procédés de dénomination est l'usage des termes à partir desquels des internautes s'auto-caractérisent. Dans ce sillage, l'on peut considérer : WISEMAN, FILS DU PAYS, LE PATRIOTE et LE PENSEUR :

[24]

\begin{abstract}
WISEMAN
desexples nous pouvons en citer a l'infini sans trouver une seule raison objective de blamer le retard economique des pays africains francophones sur le f.cfa, sans se rendre coupable de faux temoignage car la parole de dieu nous dit ceci mon père "tu ne fera point de faux temoignage...seule la veritelibere...etc."(Tournée africaine de François Hollande : un retour sibyllin aux bases militaires, MunaDimbambe, Aurore Plus,23/06/2014, www.camer.be)
\end{abstract}

FILS DU PAYS(Kerpen) GERMANY

pourquoi les africains ont si peur de la france???pouquoi les africains ont toujours une pensée à sens unique? si le sous developement de l'afrique est garant de l'industrialisation de la france, que les africains choisissent la voix qui leurs convient: soit rester sous developés au profit de la france soit se developer à tous les prix. pourquoi tous les pays habités en majorité par les blancs sont dévelopés? n'y avait-il pas aussi la une question de stratégie ou de dominance?à voir de pret les occidentaux n'ont pas attendu l'afrique pour se developer, ainsi l'afrique ne doit pas balayer les pots cassés pendant le déclin de l'occident. l'occident pourait en profiter en prenant l'afrique au sérieux...(CamerounFrance-aide au développement : la France écarte le Cameroun, Georges Alain Boyomo, Mutations, 01/08/2013, www.camer.be)

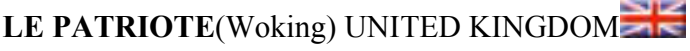

comme on pouvait le prevoir, 7 athletes camerounais on deja pris la poudre d'escampete... les autres suivrons certainement.

c'estcameme les gars... et les filles (France-Cameroun-Congo-B: Sassou et Biya malmenés par Hollande, La Lettre du Continent, 16/09/2012, www.camer.be).

[27]

LE PENSEUR (Eppertshausen) GERMANY

ilfoetre con pour croire a ce kil dit... kelk un kia cautionner 1 attake de la lybie dit vouloir rompre avec la franceafrik

dieu seul sais kan periode de disette ke traverse la france les caissse et matierepremiere africaine doit plus ke jamais etreappeller en ressource pr le bien fais des toubabs ki nous traiterons apres de tous les noms (France-Afrique : François Hollande creuse la tombe de la Françafrique, L'ouest républicain, 17/05/2012,www.camer.be)

Le premier terme provient de l'anglais. Il signifie homme intelligent. FILS DU PAYS constitue un syntagme nominal dont le complément déterminatif à la valeur d'appartenance. LE PATRIOTE est un substantif révélateur de l'amour d'un citoyen à l'égard de son pays ou sa nation. LE PENSEUR fait référence à un philosophe.

\subsection{Les termes à connotation idéologique}

Parmi les stratégies mobilisées par des internautes, nous notons des expressions à valeur idéologique. Il s'agit davantage des visions liées aux problèmes socio-politiques : 


\title{
[28]
}

\author{
FREECAMEROONNOW(Beltsville) UNITED STATES \\ paulbiyabivoleur, tricheur, margouilleur, pilleur des fonds camerounais, diviseur,baiseur \\ , traitre ...etc. \\ tu veras ta fin approche petit apetit . \\ tu tomberas avec tous ceux qui sont avec toi . \\ wait and see ! (France-Mandat d'arrêt contre le fils du président de Guinée Equatoriale, \\ Béatrice Gurrey, Lemonde.fr, 14/07/2012, www.camer.be)
}

\section{[29]}

\begin{abstract}
ABATTONLEDICTATEUR(Suresnes) FRANCE
jsais pas si certains nègrichons lisent français

on démontre bien ke le voleur-criminel-génocidaire-prédateur-usurpateur gbagbopdant 11 ans jamais élu avait surtt des pseudos-conseillers français

gbagbo pourra jamais gagner 1élection libre,transparente en civil y est très largemt minoritaire

le 1er tour des présidentielles l'a montré av son 1/3 des voix et sans réserve au 2 è tr sa propre cour consti a déclaré kil fallait supprimer 700.000voix à ado pr kil puisse gagner av

les ivoiriens de l'extérieur ont voté à $65 \% \mathrm{pr}$ ado ainsi ke $63 \%$ des militaires gbagbo a été honteusemt battu par ado ds son propre village natal à gagnoa av $59 \%$ aujprésidt ado a le droit de pdre des experts en france(gde démocratie) pr parfaire l'administration ivoirienne voire $\mathrm{mm}$ africaine au service du peuple (Côte d'Ivoire: Ouattara sous l'influence de ses sorciers blancs, La Lettre du Continent, 24/01/2012,www.camer.be)
\end{abstract}

Ces trois expressions traduisent la vision sociopolitique des internautes. En (28), le locuteur utilise l'expression FREECAMEROONNOW. Elle traduit le regard critique de cet internaute sur la gestion politique du Cameroun. Le discours qu'il mobilise le prouve suffisamment. ABATTONLEDICTATEUR (29) exprime également un positionnement critique de l'internaute. Il se présente comme un internaute qui défend l'idéal démocratique. Pour lui, tous les Présidents, à l'instar de Gbagbo, qui ne respectent pas les principes démocratiques, sont considérés comme des dictateurs. La nomination qu'il se donne révèle sa volonté de voir les présidents dictateurs de l'Afrique noire à quitter le pouvoir.

\section{La pseudonymie des internautes : quelle significativité ?}

Selon Béliard (2009 : 196), l'une des caractéristiques du pseudonyme est qu'il n'est pas reçu, il est choisi. Il n'est donc pas vécu comme un «stigmate » subi. Par son choix, l'individu anticipe les interactions et les oriente, en fonction de ce qu'il veut montrer aux autres ». On comprend que les autonymes analysés sont des formes signifiantes liées aux intentions de communication. A ce sujet, nous pouvons concevoir cinq matrices signifiantes en rapport avec le type de société virtuelle observé.

\subsection{L'expression des identités et des cultures}

L'auto-nomination des internautes camerounais par des expressions qui relèvent du domaine de la culture et de l'art constitue une stratégie efficace pour la mise en exergue des valeurs identitaires et culturelles. Ces valeurs peuvent être inhérentes à certaines entités sociales. Les indices lexicaux ainsi mobilisés sont susceptibles de refléter l'identité sociale de l'internaute. Les cas relevés tels que PA'A TAGNE (5), NKUNKUMA 2 (6) et 
BETIBENANGA (8) drainent des identités sociales des régions de l'Ouest et du Centre du Cameroun. Mais il peut arriver que ces marqueurs ne correspondent pas toujours à l'identité réelle de l'internaute.

Sur le plan culturel, nous avons des indicateurs qui montrent que les internautes voudraient revitaliser certaines valeurs. C'est ce qui pourrait justifier les marqueurs tels que MAN TARA (8) et MATANGO (9) qui sont relatifs à une praxis linguistique et à un domaine viticole. Par ces indicateurs, l'on voit en filigrane, l'identité ewondo, une composante tribale inhérente à la région du Centre.

\subsection{La commémoration des stars du football, de la musique, de la philosophie et de la mythologie}

Certains pseudonymes adoptés par des internautes sont au service de la revitalisation des icônes du football mondial et de la musique camerounaise. Dans le corpus, nous avons analysé les pseudonymes issus des noms des footballeurs qui ont marqué le sport à une époque donnée. C'est ce qui expliquerait les noms suivants: ARANTES (10), BEBETO (11), ZANGALEWA (12) et WANGRIN (13). L'on peut présumer que ces vedettes constituent, pour ces internautes, des modèles du football camerounais et brésilien, de la musique camerounaise et la littérature malienne. Pour manifester leur célébrité, leurs noms deviennent des pseudonymes. C'est dans cette même perspective qu'on l'on peut interpréter les noms renvoyant aux philosophes et à la mythologie grecque : CLEOPATRE (17), PLATON (18) et EURYALES (19). Leur usage est consécutif à deux intentions de communications : soit les internautes voudraient marquer leur compétence en matière de la philosophie et de mythe, soit ils aimeraient incarner les idées de ces penseurs ou les idéaux des mythes. Dans tous les cas, ils ont une fonction identificatoire, puisqu'ils «devraient idéalement permettre à celui ou celle qui s'en sert de s'approprier les qualités d'une personne admirée via l'utilisation de son nom. Il s'agit souvent de personnages célèbres ou d'artistes » (Maribel Fehlmann, 2010 : 268). En s'autonomant PLATON ou WANGRIN, l'internaute "pourrait se voir paré-e des qualités/pouvoirs exceptionnels évoqués par le nom choisi, qu'il s'agisse de propriétés évoquées par le nom lui-même » (Maribel Fehlmann, $2010: 267$ ) d'où leur fonction encomiastique. Ces propriétés sont généralement de l'ordre du désirable.

\subsection{L'expression de la mémoire des territoires}

Il est n'est exclu de considérer l'usage de certains pseudonymes comme une stratégie consistant à mettre sur la toile la mémoire des territoires du Cameroun. Ces espaces sont des quartiers ou des villes : NDOKOTI-MVOG MBI (12), DEIDO (14), MVOG ADA (15) et KOLOFATA (16). L'option pour ces toponymes n'est pas sans motivation. En effet, nous avons affaire à des noms des lieux urbains qui ont des connotations sociales particulières. NDOKOTI-MVOG MBI, DEIDO et MVOG ADA sont des quartiers des deux grandes villes du Cameroun. Ils peuvent être taxés de quartiers pauvres et dangereux, caractérisés par une surpopulation, le désordre urbain, la pauvreté et de nombreuses activités commerciales qui échappent parfois au contrôle de l'Etat. Leur adoption par certains internautes se lit comme une forme de sympathie, voire d'empathie qu'ils voudraient manifester à l'endroit des occupants de ces quartiers populeux. Par ailleurs, le cas de KOLOFATA est très significatif, dans la mesure où ce nom réfère à une localité de la région de l'Extrême-Nord du Cameroun qui subit les actes terroristes les plus meurtrières $\mathrm{du}$ pays. Utiliser ce nom comme pseudonyme devient une manière subtile de rendre 
hommage à tous ceux qui ont perdu leur vie dans cette localité lors des attentats et surtout de tenter de garder en mémoire les exactions terroristes commises.

\subsection{Le marquage des positionnements sociopolitiques}

L'un des enjeux des pseudonymes utilisés par des internautes est l'expression des positionnements sociopolitiques. A travers certains noms utililisés, l'on perçoit les visions politiques des internautes. Dans ce sillage, il convient de reconsidérer les cas suivants : EPERVIER (20), RDPC FOREVER (21), BIYABIVOLEUR (22), FREECAMEROONNOW (28) et ABATTONLEDICTATEUR (29). Ces nominations entrent dans les discours stéréotypés qui circulent au Cameroun. EPERVIER utilisé par l'internaute, nous l'avons dit, rappelle une opération judiciaire, initiée en 2006 par le gouvernement du Cameroun, pour lutter contre la corruption. RDPC FOREVER montre l'engagement sincère ou ironique de l'internaute à adhérer au Rassemblement démocratique $\mathrm{du}$ peuple camerounais. BIYABIVOLEUR dénote l'antipathie ou l'opposition de l'internaute au régime du Président de la République du Cameroun, son excellence Paul Biya. FREECAMEROONNOW et ABATTONLEDICTATEUR reflètent la thèse selon laquelle l'Afrique en général et le Cameroun en particulier sont sous le joug de la néocolonisation, appuyée par la complicité des dirigeants africains.

Les discours émis par ces internautes révèlent leurs convictions sociopolitiques. C'est ce qui amène Martin (2013 : 786) à dire que «sur les forums, certains usagers donnent à leur pseudonyme un tour politique, voire revendicatif, où, par le biais d'un «nom fait à plaisir », ils marquent une prise de position ou encore ils émettent opinions et sentiments sur des situations données $»$.

\subsection{L'expression de l'image de soi}

Certains pseudonymes utilisés par des internautes contribuent à exprimer une image personnelle qu'ils voudraient afficher. Cette image permet de voir l'idée que l'internaute se fait de lui-même. Son objectif est alors de l'extérioriser. Dans cette optique, nous avons : WISEMAN (24), LE PENSEUR (27), FILS DU PAYS (25) et LE PATRIOTE (26). Les deux premiers termes permettent aux internautes de se présenter comme des hommes sages et éclairés, capables de produire des thèses pertinentes dans le forum de discussion. FILS DU PAYS et LE PATRIOTE montrent que ces internautes voudraient se caractériser par leur amour du pays. Ils ont intérêt à défendre l'intégrité de leur nation. C'est cet élan de patriotisme qui les pousse à critiquer les politiques gouvernementales des dirigeant africains, à remettre en question la coopération entre la France et l'Afrique. Ces internautes projettent donc ce qu'Amossy (2000:9) appelle " image de soi », appréhendée comme une présentation de soi que le locuteur effectue dans son discours. Il ressort que les pseudonymes de cette catégorie reflètent des images subjectives des internautes. En d'autres termes, il s'agit de l'expression d'un moi qui se veut singulier dans le forum de discussion. C'est pourquoi Georgeta Cislaru (2009) dit que « le pseudonyme est condamné à signifier [...]. Sa signifiance implique directement l'individu-sujet et contribue à la construction de son ethos discursif $»$. Son enjeu primordial, contrairement au patronyme ou au nom propre de personne, est « l'intention de communiquer sur soi ». L'individu-sujet est donc intrinsèquement lié au pseudonyme qu'il a choisi. Dès lors, il est « différent du nom et de tous les autres identifiants : il est personnel ; ceci implique qu'il reflète directement ou intrinsèquement une caractéristique de la personnalité de son porteur » (Khaled Slama, 
2011 : 11). Il peut être conçu en fonction de l'histoire du blogueur, de son entourage, ses penchants, ses désirs ou ses visions personnelles.

\section{Conclusion}

Cette réflexion s'est appesantie sur les processus d'auto-nomination des internautes participant à certains forums de discussion animés par des Camerounais ou ceux qui s'intéressent aux problèmes du Cameroun. A ce sujet, nous avons analysé plusieurs constructions effectuées par ces derniers. Les lieux sources des pseudonymes les plus saillants sont: l'identité (culture, tribu), le football, la patronymie, l'art, les références sociopolitiques et les marqueurs de la subjectivité. C'est cet ensemble qui structure la pseudonymie mise en œuvre par les internautes camerounais. L'examen de ces mécanismes conduit à la saisie de leurs enjeux, puisque leur actualisation est motivée. Dans cette optique, la portée de ces procédés est de plusieurs ordres : la valorisation des identités sociales et artistiques, l'expression de la mémoire des territoires significatifs, les postures socio-idéologiques et la projection de l'image de soi. Au bout du compte, on peut dire que les constructions des pseudonymes dans le terrain virtuel camerounais ne sont pas une simple stratégie de voiler son identité authentique afin d'éviter la censure juridique en cas de dérapage ou d'atteinte aux droit d'autrui.

Ces pseudonymes sont porteurs des significations liées aux phénomènes sociaux qui caractérisent le Cameroun. La pseudonymie devient ainsi un facteur d'un quasi genre de discours sur Internet, notamment les discours diasporiques et ceux qui sont produits localement. Au regard des mécanismes pseudonymiques examinés, ce genre de discours traduit la volonté de ses producteurs d'actionner les valeurs identitaires et culturelles. De même, l'occasion est offerte aux Camerounais de traduire leur vision socio-politique ou du moins, leur point de vue sur la gestion de la nation camerounaise. Internet, terrain de recherche complexe devient ainsi un espace de dialogue social où l'identité réelle des participants n'est pas explicite, mais implicite, puisque les pseudonymes employés permettent de saisir leurs différents positionnements sociaux.

\section{Références bibliographiques}

Amossy, R (dir.). (200). Introduction, Images de soi dans le discours. La construction de l'ethos, Lausanne : Delachaux et Niestlé, pp.930.

Anis, J. (2001). Approche sémiolinguistique des représentations de l'ego dans la communication médiée par ordinateur. Psycholinguistique et intelligence artificielle, Langages, $\mathrm{N}^{\circ} 144$, pp.20-38.

Barry, A. O. (2002). Les bases théoriques en analyse du discours, Les textes de méthodologie, [en ligne], consulté le 23 novembre 2017, https///depot.erudit.org/id/002331do.

Béliard, A-S. (2009), Pseudos, avatars et bannières : la mise en scène des fans de la série télévisée Prison Break (enquête), Terrains \& travaux, N¹5, pp.191-212.

Cislaru, G. (2011). Le pseudonyme, nom ou discours ? D'Etienne Platon à Oxyhre, Les Carnets $d u$ Cediscor, $\mathrm{N}^{\circ} 11$, [en ligne], consulté le 01 octobre 2016, URL : http://cediscor.revues.org/746.

Fehlmann, M. (2010). De la valeur magique du pseudonyme sur internet. Nouvelle revue d'onomastique, $\mathrm{N}^{\circ} 52$, pp.263-275. 
Guthrie, M. (1958/1967). The Classification of the Bantu Languages, London, Dawsons of Pall Mall for IAI.

Khaled, S. S. (2011). L'usage du pseudonyme et éthique sur Internet. Questions éthiques, Revue Pertinence, $\mathrm{N}^{\circ} 4$, Archives ouvertes.

Maingueneau, D. (2005). L’analyse du discours et ses frontières. Marges linguistiques, $\mathrm{N}^{\circ} 9$, pp.6475.

Maingueneau, D. (2012). Que cherchent les analystes du discours?, Argumentation et analyse du discours, [en ligne], $\mathrm{N}^{\circ}$, consulté le 30 septembre 2016, http://aad.revue.org/1354.

Martin, M. (2013), La vocation plurielle du pseudonyme sur Internet, BDD-V1546, Editura Maga, EdituraArgonaut, pp.785-792.

Pierozak, I. (2007). Prendre Internet pour terrain. Glottopol, [en ligne], $\mathrm{N}^{\circ} 10$, http://www.univrouen.fr/dyalang/glottopol, pp.4-10.

Siblot, P., (1997). Nomination et production de sens : le praxème. Langages, $\mathrm{N}^{\circ} 127$, pp. 38-55.

Siblot, P., (2001). De la dénomination à la nomination. Cahiers de praxématique [En ligne], №36, document 8, mis en ligne le 01 janvier 2009, consulté le 30 septembre 2016. URL :

http://praxematique.revues.org/368.

Siblot, P., (2006). Problématique de la nomination : du répertoire des sens à l'analyse de leur production. Neologica, $\mathrm{N}^{\circ} 1,2006, \mathrm{pp} .35-50$.

www.forumbonaberi.com

www.camer.be

www.cameroon.info.net 